\title{
Erratum to: Depression, anxiety, and quality of life in a large cohort of patients with rheumatic diseases: common, yet undertreated
}

\author{
Panagiota Anyfanti $^{1}$ - Eleni Gavriilaki ${ }^{2}$ Athina Pyrpasopoulou ${ }^{1}$. \\ George Triantafyllou $^{2} \cdot$ Areti Triantafyllou $^{2} \cdot$ Sofia Chatzimichailidou $^{1}$. \\ Eugenia Gkaliagkousi ${ }^{1} \cdot$ Spyros Aslanidis $^{1} \cdot$ Stella Douma $^{2}$
}

Published online: 1 April 2016

(C) International League of Associations for Rheumatology (ILAR) 2016

Erratum to: Clin Rheumatol (2016) 35:733-739

DOI 10.1007/s10067-014-2677-0

The original version of this article unfortunately contains an error in the Abstract section. In particular, it was mentioned by mistake that prevalence of anxiety is $30.8 \%$, which is incorrect. The prevalence of anxiety should be $37 \%$, as pointed in page 735 both in the Results text and Table 2 .

Therefore, the correct sentence (10th-12th line of the Abstract section from the top) should now be:

"Depression and anxiety were documented in 21.8 and $37 \%$ of the population, respectively, and correlated significantly with quality of life."

The online version of the original article can be found at http://dx.doi.org/ 10.1007/s10067-014-2677-0.

Eleni Gavriilaki

elenicelli@yahoo.gr

1 2nd Propedeutic Department of Internal Medicine, Aristotle University of Thessaloniki, Thessaloniki, Greece

2 3rd Department of Internal Medicine, Papageorgiou Hospital, Aristotle University of Thessaloniki, Ring Road Nea Eukarpia, 56403 Thessaloniki, Greece 